\title{
Emergence of Symmetry in Complex Networks
}

\author{
Yanghua Xiao $^{1}$, Momiao Xiong ${ }^{2,3}$, Wei Wang ${ }^{1}$, and Hui Wang ${ }^{4}$
}

\begin{abstract}
${ }^{1}$ Department of Computing and Information Technology, Fudan University, Shanghai 200433, PR China
${ }^{2}$ Theoretical Systems Biology Lab , School of Life Science, Fudan University, Shanghai 200433, PR China ${ }^{3}$ Human Genetics Center, University of Texas Health Science Center at Houston, Houston TX 77225, USA and ${ }^{4}$ Business School, University of ShangHai for Science and Technology, Shanghai 200093, PR China
\end{abstract}

(Dated: November 12, 2018)

\begin{abstract}
Many real networks have been found to have a rich degree of symmetry, which is a very important structural property of complex network, yet has been rarely studied so far. And where does symmetry comes from has not been explained. To explore the mechanism underlying symmetry of the networks, we studied statistics of certain local symmetric motifs, such as symmetric bicliques and generalized symmetric bicliques, which contribute to local symmetry of networks. We found that symmetry of complex networks is a consequence of similar linkage pattern, which means that nodes with similar degree tend to share similar linkage targets. A improved version of BA model integrating similar linkage pattern successfully reproduces the symmetry of real networks, indicating that similar linkage pattern is the underlying ingredient that responsible for the emergence of the symmetry in complex networks.
\end{abstract}

PACS numbers: 89.75.-k 89.75.Fb 05.40.-a 02.20.-a

In the last decades, we have witnessed the great progress in the complex network researches $11,2,3,4$, 5, 6, 7, 8, 9, 10, 11, 12, 13, 14]. Previous studies have primarily focused on finding the statistical properties of various networks, such as small world property $[\underline{3}$, 5, 8]; power-law distribution of vertex degree [4]; building block of network motifs [9]; assortative mixing [12]; self-similarity[6]; community structure [10, 11, 13] and hierarchical structure 14] of the network. And based on these properties, many network models, such as Barabaśi-Albert (BA) [4] model, Watts-Strogatz model [8] have been proposed to help predict the future evolution of the network. However, an important property of network structure, symmetry, has been rarely studied.

Concept of symmetry is based on the concept of automorphism of the graph, which characterizes adjacency invariance to transformation operation on the node set. Graph has been widely used to represent systems consisting of components (represented by nodes) as well as their relation (represented by edges). If two nodes are connected by an edge they are defined as adjacent nodes. An automorphism acting on the node set can be viewed as a permutation of the nodes of the graph preserving the adjacency of the nodes. The set of automorphisms under the product of permutation forms a group 21]. In general, a network is considered as asymmetric if its underlying graph contains only an identity permutation, otherwise, the network is symmetric.

It has bee shown that various complex networks have a rich degree of symmetry 22, 23]. The fact that large real networks are symmetric is surprising 22], since 'almost all graphs are asymmetric' 26] [28]. As an ubiquitous phenomenon, the existence of symmetry in the real networks strongly begs an explanation, since existing ingredients, such as continuous growth and preferential attachment [4] dominating the construction of the network structure, are not dedicated to interpret the origination of symmetry in real networks.

To explore the origin of symmetry in real networks, we summarize statistics of the local symmetric motifs contributing to the symmetry of the real networks, by which we found that similar linkage pattern[29], which means that nodes having similar property, for example degree, tend to have similar linkage targets, is a ubiquitous law that dominating the construction of structures of a variety of real networks. For example, in a friendship network, it is widely believed that persons with similar properties such as educational background, interest, age, would probably have common friends.

To show that similar linkage pattern is a ubiquitous law that holds across many structures of real networks, we first summarize the statistics of symmetric bicliques in the real networks, which is a induced complete bipartite subgraph, denoted as $K_{V_{1}, V_{2}}$, in which the degree of vertices in $V_{1}$ is conserved [30]. Obviously, if graph $G$ contains a symmetric biclique $K_{V_{1}, V_{2}}$, then the automorphism group of $G$, denoted as $\operatorname{Aut}(G)$, will have a corresponding geometric decomposition factor 23] $S_{n}$ with $n=\left|V_{1}\right|$, which indicates that the size of $\operatorname{Aut}(G)$ has a factor of $n$ !. Thus, $K_{V_{1}, V_{2}}$ becomes a local symmetric motifs [23] contributing to the symmetry of the network. Hence, symmetric bicliques will contribute to the symmetry of the network. Figure1 1 illustrate two such bicliques.

If we do not care about what $V_{1}$ and $V_{2}$ are, we also use $K_{i, j}$ to denote $K_{V_{1}, V_{2}}$, where $\left|V_{1}\right|=i$ and $\left|V_{j}\right|=j$. And the set consisting of all $K_{i, j}$ is denoted as $\mathcal{K}_{i, j}$. Note that $\mathcal{K}_{1, i}$ does not necessarily contribute to the local symmetry of the network, hence, in the following discussion, 


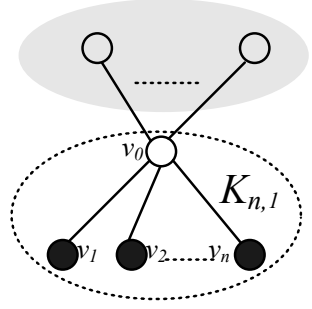

(a)

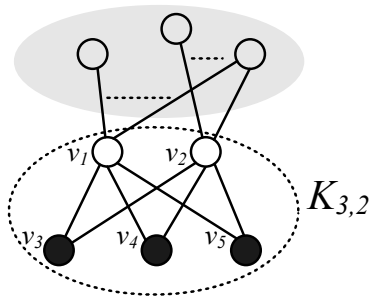

(b)
FIG. 1: Illustration of symmetric bicliques. Figure (a) shows an example of $K_{n, 1}$, which contributes to the symmetry of the network with a geometric decomposition factor $n$ !; figure (b) shows an example of $K_{3,2}$, which contributes to the symmetry of the network with a geometric decomposition factor $S_{3}$.
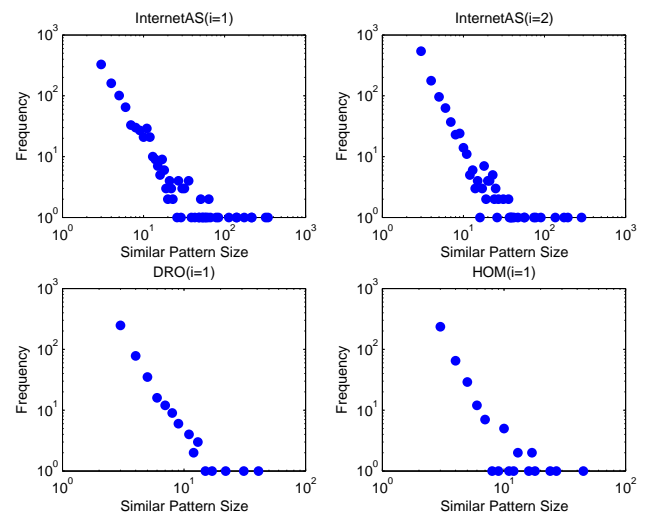

FIG. 2: Size distribution of the symmetric bicliques for real networks. The horizontal axis for each panel is the size of symmetric bicliques and the vertical axis is the occurrence frequency of the symmetric bicliques with the corresponding size. Figure (a) and (b) show the biclique size distribution of the Internet at autonomous level $\square$ for $\mathcal{K}_{n, 1}$ and $\mathcal{K}_{n, 2}$ respectively; Figure (c) shows the biclique size distribution of $\mathcal{K}_{n, 1}$ of Homo Sapiens [20]. Figure (d) shows biclique size distribution of $\mathcal{K}_{n, 1}$ of Drosophila melanogaster [20].

${ }^{a}$ Here, the snapshot at 2006-07-10 of CAIDA 18] is used.

only $\mathcal{K}_{n, i}$ with $n \geq 2$ has been summarized.

As shown in Table 【, similar linkage pattern is a universe phenomenon in the process of structure construction of many real networks including social networks, biological networks and technological networks. For instance, for $\mathcal{K}_{n, 1}$ on Internet $A S$ dataset, there are totally 916 non-disjoint 31] structures, among which there exists some larger symmetric motifs, e.g. the maximal motif has 343 nodes in $V_{1}$. For all the network we tested, simple symmetric motifs such as $\mathcal{K}_{n, 1}$ and $\mathcal{K}_{n, 2}$ can be frequently observed. Moreover, for some networks, such as biogrid network 'DRO', even for some larger $i$, more complex symmetric motifs of $\mathcal{K}_{n, i}$ do exist.

As shown in Figure 2 among those simple symmetric motifs with $i=1,2$, the size (n) distributions are rightskewed with a long tail for larger size, which implies that a number of larger patterns do exist.

Furthermore, we will show that similar linkage pattern will not happen in ER 27 random graphs. As shown in Table [II] only few randomized networks having the same size as the corresponding real networks, have symmetric motifs of $\mathcal{K}_{n, 1}$; and no larger motifs $\mathcal{K}_{n, i}$ with $i \geq 2$ exist. Also we found that the number of motifs in $\mathcal{K}_{n, 1}$ of randomized networks is much less than that of the corresponding real networks with the same size, the complexity of the motifs are much lower than that of the corresponding real networks.

The frequent occurrence of complex $K_{n, i}$ in real networks and the unfrequent occurrence of complex $K_{n, i}$ in random networks strongly suggest that there exist some laws dominating the structure construction process of real networks. Consider the dynamic process of the network growth. We assume that at some time a new node $v$ is added to the network, and a symmetric motif $K_{V_{1}, V_{2}}$ will arise. Thus, from the facts we have observed, it's reasonable to believe that $v$ will attach to the existing nodes under the principle of preferentially linking to those nodes to which other nodes in $V_{1}$ attach. Since nodes in $V_{1}$ have the same degree, it's reasonable to believe that nodes having the same degree will have the same linkage pattern.

However, as shown in Figure 3, in real networks, nodes having the same degree tend to have only similar targets not exactly the same target sets, and these local motifs exhibiting non-exact similar linkage behavior also have chance to contribute to the symmetry of the network. Clearly, these local substructures are the generalization of symmetric bicliques, in the way that structures constraint of the clique is relaxed from being complete bipartite to only satisfying that all the vertex of $V_{1}$ have the same degree in the clique. Thus, this kind of generalized symmetric biclique can be denoted as $K_{V_{1}, V_{2}}^{d}$, where $d$ is the degree of any vertex in $V_{1}$; in some cases that we do not care about $V_{2}, K_{V_{1}}^{d}$ or $K_{\left|V_{1}\right|}^{d}$ is often used.

In a network, if non-exact similar linkage pattern does make sense, then nodes with the same degree will tend to share similar linkage targets. Thus, we need to measure in what extent these nodes share the same targets. Let $V(m)=\{v: v \in V$ and $d(v)=m\}$ be all nodes with degree $m$, then the linkage targets of these nodes could be denoted as $V(m)^{\prime}=\left\{v^{\prime}:\left(v, v^{\prime}\right) \in E\right.$ and $\left.v \in V(m)\right\}$. Then we could define $\theta_{m}$ as Equation 1, which is the ratio of the actual number of linkage targets of $V(m)$ to the maximal probable number of linkage targets( Maximal set of linkage targets could be obtained when overlapping of targets of nodes in $V(m)$ is forbidden.) Obviously, this measure is a key index that can be used to quantify overlapping ratio of linkage target of nodes in $V(m)$.

$$
\theta_{m}=\frac{\left|V^{\prime}(m)\right|}{m|V(m)|}
$$

Obviously, we have $0<\theta_{m} \leq 1$. If $\left|V_{m}\right|$ is given, 
TABLE I: Symmetric biclique statistics of a number of real networks. We measure the size of the networks by the number of nodes and edges, denoted by $\mathrm{N}$ and $\mathrm{M}$, respectively. For each $i \leq 7$, the statistics of symmetric bicliques contained in $\mathcal{K}_{n, i}$ with $n \geq 2$ is measured. We use a triple tuple $(S, \operatorname{Min}, \operatorname{Max})$ to show the statistics of $\mathcal{K}_{n, i}$, where $S$ is the number of non-equal substructures in $\mathcal{K}_{n, i}$, and the $\operatorname{Min}, \operatorname{Max}$ are the minimal and maximal size of symmetric bicliques, respectively. If $\mathcal{K}_{n, i}$ is empty, $S=0$, and Min and Max is not available, denoted as '-'. For some larger $i$, we also enumerate corresponding statistics of $\mathcal{K}_{n, i}$.

\begin{tabular}{|c|c|c|c|c|c|c|c|c|}
\hline \multirow[b]{2}{*}{ Network } & \multicolumn{8}{|c|}{$\mathcal{K}_{n, i}$ with $n \geq 2$} \\
\hline & 1 & 2 & 3 & 4 & 5 & 6 & 7 & some larger i \\
\hline $\operatorname{arXiv}[19]^{a}$ & $(135,2,7)$ & $(42,2,4)$ & $(17,2,3)$ & $(13,2,2)$ & $(11,2,2)$ & $(1,2,2)$ & $(2,2,2)$ & $i=16,(1,2,2)$ \\
\hline InternetAS ${ }^{b}$ & $(916,2,343)$ & $(1057,2,285)$ & $(90,2,25)$ & $(9,2,4)$ & $(2,2,2)$ & $(0,-,-)$ & $(0,-,-)$ & $(0,-,-)$ \\
\hline $\mathrm{SAC}$ & $(51,2,15)$ & $(7,2,5)$ & $(0,-,-)$ & $(0,-,-)$ & $(0,-,-)$ & $(0,-,-)$ & $(0,-,-)$ & $(0,-,-)$ \\
\hline MUS & $(7,2,44)$ & $(8,2,12)$ & $(4,2,6)$ & $(2,2,2)$ & $(0,-,-)$ & $(1,2,2)$ & $(0,-,-)$ & $(0,-,-)$ \\
\hline $\mathrm{HOM}$ & $(366,2,44)$ & $(53,2,12)$ & $(21,2,6)$ & $(5,2,2)$ & $(2,2,2)$ & $(1,2,2)$ & $(0,-,-)$ & $i=8,10,21,(1,2,2)$ \\
\hline $\mathrm{CAE}$ & $(245,2,47)$ & $(9,2,5)$ & $(1,2,2)$ & $(0,-,-)$ & $(0,-,-)$ & $(0,-,-)$ & $(0,-,-)$ & $(0,-,-)$ \\
\hline USPowerGrid[8] & $(137,2,9)$ & $(25,2,3)$ & $(0,-,-)$ & $(1,2,2)$ & $(0,-,-)$ & $(0,-,-)$ & $(0,-,-)$ & $(0,-,-)$ \\
\hline
\end{tabular}

${ }^{a}$ Here, the snapshot at 2006-03 of HEP-TH (high energy physics theory) citation graph [19] is used.

${ }^{b}$ Here, the snapshot at 2006-07-10 of CAIDA [18] is used.

TABLE II: Symmetry biclique statistics for a variety of ER random networks. For each network tested in Table [ we generate the corresponding ER random networks with the same size using PAJEK [24]. Two parameters are needed, the vertex number $N$ and the average degree $z$ to ensure the generated random network having the same size with the corresponding real networks. Similar to the evaluation in Table I we also measure the statistics for each $\mathcal{K}_{n, i}$, but only statistics of $\mathcal{K}_{n, 1}$ is available for some random networks. Larger symmetric bicliques $\mathcal{K}_{n, i}$ with larger $i$, do not exist in these random networks.

\begin{tabular}{cccc}
\hline \hline Network & $\mathrm{N}$ & $\mathrm{z}$ & $\mathcal{K}_{n, 1}$ with $n \geq 2$ \\
\hline arXiv & 27770 & 25.37 & $(0,-,-)$ \\
InternetAS & 22442 & 4.06 & $(62,2,3)$ \\
BioGrid & & & \\
SAC & 5437 & 26.86 & $(0,-,-)$ \\
MUS & 218 & 3.65 & $(1,2,2)$ \\
HOM & 7522 & 5.32 & $(3,2,2)$ \\
DRO & 7528 & 6.69 & $(0,-,-)$ \\
CAE & 2780 & 3.13 & $(21,2,3)$ \\
USPowerGrid & 4941 & 1.49 & $(231,2,3)$ \\
\hline \hline
\end{tabular}

we have $\frac{1}{V(m)} \leq \theta_{m} \leq 1$. Note that, the lower $\theta_{m}$ is, the more frequently similar linkage pattern will happen, while for $\theta_{m}$ 's closer to 1 , the exact opposite is true. As shown in Figure 4, for small values of degrees, all tested networks tend to have relative small $\theta_{m}$, which strongly suggest that for these real networks, in the process of network growth, nodes with the same small degree tend to have the similar linkage behavior.

It has been shown in the BA[4] model that many real networks have power law degree distribution, which can be attributed to two basic ingredients: (1) Growth and (2)Preferential attachment. In BA model, new nodes will

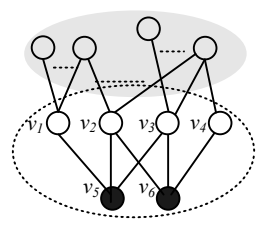

(a)

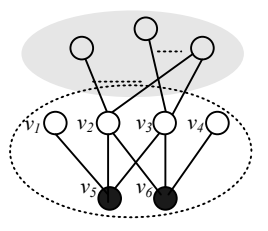

(b)

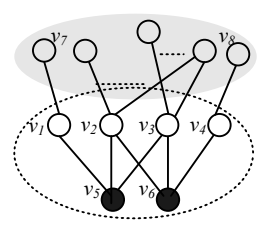

(c)
FIG. 3: Illustration of non-exact similar linkage pattern. All nodes in $V_{1}$, i.e., dark nodes, have the same number of linkages. However, the linkage target of these nodes are not exactly the same. Motifs induced by $V_{1}$ as well as their linkage targets, do not necessarily contribute to the local symmetry of networks. In Figure 3(a), the subgraph induced by $V=\left\{v_{1}, \ldots, v_{6}\right\}$ will not lead to any automorphism, while the induced subgraph in Figure 3(b) will result in an automorphism $p=\left(v_{1}, v_{4}\right)\left(v_{5}, v_{6}\right)$ and the subgraph induced by $V=\left\{v_{1}, \ldots, v_{8}\right\}$ in Figure $3(\mathrm{c})$ also contributes an automorphism $p=\left(v_{1}, v_{4}\right)\left(v_{5}, v_{6}\right)\left(v_{7}, v_{8}\right)$ to the symmetry of the graph.

be continuously added to the existing networks, and at each time step, a new node is preferentially attached to fixed number of $m$ (this number $m$ is referred to as initial degree of the newly added node) existing highly connected nodes. However, ingredients about symmetry have not been considered in BA model and other network generation models. To reproduce symmetric networks with power law degree distribution, we propose a new network model incorporating similar linkage pattern into BA's two ingredients. For this purpose, two modifications on BA model's two principles need to be carried out:

1. Newly added nodes are linked to the existing nodes not only under the principle of preferential attach- 

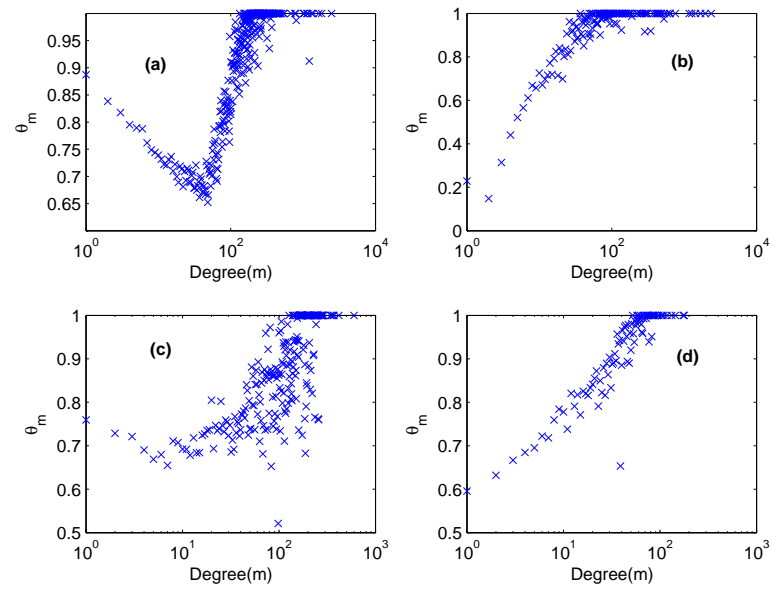

FIG. 4: Figure(a),(b),(c),(d) show $\theta_{m}$ distribution of arXiv, Internet, Biogrid SAC and Biogrid DRO, respectively.

ment, but also similar linkage pattern. The latter principle implies that newly added node with initial degree $m$ tends to link to the targets to which existing nodes with degree $m$ in the network are linked.

2. Initial degree $m$ of newly added nodes follows a certain distribution instead of being a constant value. In BA and other existing models, initial degree is constant, while in the following study, we will show that in many real networks, $m$ follows a certain distribution.

The probability, denoted by $\Pi$, that a new node with initial degree $m$ will be connected to node $v_{i}$ not only rely on degree $k_{i}$ of node $v_{i}$ but also depends on whether $v_{i}$ belongs to $V_{t}^{\prime}(m)$. To incorporate the ingredient of similar linkage pattern into the basic BA model, we need to increase $\Pi\left(v_{i}\right)$ for those $v_{i}$ belonging to $V_{t}^{\prime}(m)$. Hence, we define parameter $\alpha$ to control the relative significance of similar linkage pattern in the formation of network structure. Note that for a given $m, V_{t}^{\prime}(m)$ is not necessarily to be non-empty, hence the probability $\Pi$ would be defined in two cases: when $V_{t}^{\prime}(m)=\emptyset$, then $\Pi$ should be defined as:

$$
\Pi\left(v_{i}\right)=\frac{k_{i}}{\sum_{j} k_{j}} .
$$

where $k_{i}$ is the degree of vertex $v_{i}$; when $V_{t}^{\prime}(m) \neq \emptyset, \Pi$ should be defined as :

$$
\Pi\left(v_{i}\right)= \begin{cases}\alpha \frac{k_{i}}{\sum_{j} k_{j}}+(1-\alpha) \frac{1}{\left|V_{t}^{\prime}(m)\right|} & \text { if } v_{i} \in V_{t}^{\prime}(m), \\ \alpha \frac{k_{i}}{\sum_{j} k_{j}} & \text { if } v_{i} \notin V_{t}^{\prime}(m) .\end{cases}
$$

, where $\alpha \in(0,1]$.

At some time step $t$, we may have $V_{t}^{\prime}(m)=\emptyset$ and $\Pi$ would be reduced to pure preferential attachment in

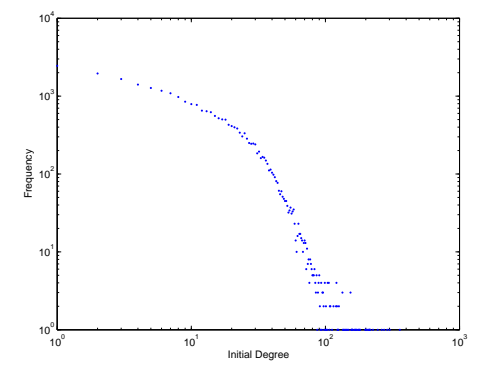

FIG. 5: Distribution of initial degree of arXiv dataset

terms of the value of degree. It will happen frequently in the initial stage of the network growth in our model. Because the abundance of degree is limited in the initial stage, whatever the seed network is. For example, if the seed network contains isolated vertices, only degree 0 could be found, if the seed network is an regular network, such as a complete network, we could also find only one degree in the network.

Equation 3 has only one parameter $\alpha$ to control the relative significance of pure preferential attachment and similar linkage pattern. It's clear that the larger $\alpha$ is, the less impact of similar linkage pattern on the network will be. When $\alpha=1$, the model is reduced to the pure preferential attachment according to vertex degree.

Obviously,the basic assumption of BA model is that any node except for those seed nodes has the same initial degree. However, for some networks, especially social networks and technique networks, whose historical data about initial degree of real networks is available, we can easily find that the initial degree of real networks may be far away from a fixed value or a value independent of degree. For example, Figure 5 shows the distribution of initial degree of a citation network constructed from arXiv data set. From this figure, we can see that for larger initial degree, the frequency follows power law distribution rather than a fixed value.

Assume that we grows the network in the way following the principle of preferentially attachment with similar linkage pattern. If the initial degree is constant, then each time a new node was added to the network, fixed number $(m)$ edges would be introduced into the network. Thus, the local symmetric motifs would concentrate on those subgraphs with structure closer to $K_{i}^{m}$. If $m$ is very larger than 1, it's contradictory to the above observed fact that the larger $n$ is, the less frequently $K_{i}^{n}$ tends to occur.

Hence, it is necessary to extend the initial degree from a fixed value to some given distribution. From this perspective, BA model would be considered as a special case of our model in the way that $F(m)$ is specified as a constant value.

The algorithm of the model incorporating the ingredi- 
ent of similar linkage pattern is the following:

(1) Growth: Starting from a small number $\left(n_{0}\right)$ of isolated nodes, at every time step, we add a new node with $m$ edges that link the new node to $m$ different nodes already present in the system, where $m$ follows a distribution $F(m)$ and $m \leq \bar{m}$, where $\bar{m}$ is the upper bound of the initial degree $m$.

(2)Preferential attachment with similar linkage pattern: The probability $\Pi$ that a new node will be connected to node $v_{i}$ is defined by Equation 2 and 3 ,

The above improved model based on similar linkage pattern needs just three input parameters $\left(n_{0}, F(m), \alpha\right)$. For the notational convenience, the model is doted as $\operatorname{SLP}\left(n_{0}, F(m), \alpha\right)$, where 'SLP' is the abbreviation of 'Similar Linkage Pattern'.

To test the effect of similar linkage pattern on symmetry of the networks, we first give some measures of symmetry of networks. The degree of the symmetry of a graph usually could be quantified by $\alpha_{G}=|A u t(G)|[25]$, i.e., the size of the automorphism group. In order to compare the symmetry of networks with different sizes, $\beta_{G}$ has been used to measure the symmetry in [22], which is defined as:

$$
\beta_{G}=\left(\alpha_{G} / N !\right)^{1 / N}
$$

, where $N$ is the number nodes in the network. $\beta_{G}$ measures the symmetry relative to maximal possible automorphism group of a graph with $N$ nodes. Another symmetry measure $\gamma_{G}$ is also given, which is the ratio of number of nodes in all those nontrivial orbits 32] (a set of equivalents nodes under automorphism operation) to the number of all nodes in the network. Specifically, let $\mathcal{P}=\left\{V_{1}, V_{2}, \ldots, V_{k}\right\}$ be the automorphism partition under the action of $(A u t(G), V)$ on node set $V, \gamma_{G}$ could be defined as :

$$
\gamma_{G}=\frac{\sum_{1 \leq i \leq k,\left|V_{i}\right|>1}\left|V_{i}\right|}{N}
$$

As shown in Figure 6(a), with $\alpha$ varying from 1 to 0.1 , i.e., with more influence resulting from similar linkage pattern exserted on the network construction, the automorphism group size of networks increase several hundreds of orders of magnitudes. The inset (a) and (b) of Figure 6(a) also show that another two symmetry indices $\beta_{G}$ and $\gamma_{G}$ increase with the decrease of $\alpha$. Such facts also can be observed from the Figure 6(b) 6(c) and 6(d) Hence, it's reasonable to believe that similar linkage pattern is responsible for the emergence of the symmetry in the networks.

If we remove the ingredient of similar linkage pattern, we will find that ingredient of preferential attachment with initial degree following a distribution will not necessarily reproduce symmetry of networks.

As shown in Figure 7, when average degree $\langle k\rangle[33$ is small (close to 1), the network have higher degree of symmetry. Note that those networks with $\langle k\rangle$ closer to 1 tend

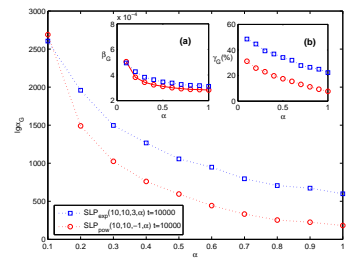

(a)

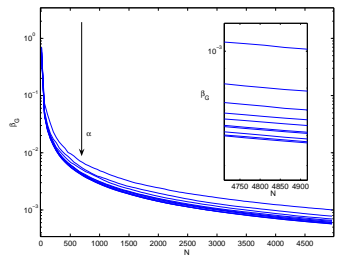

(c)

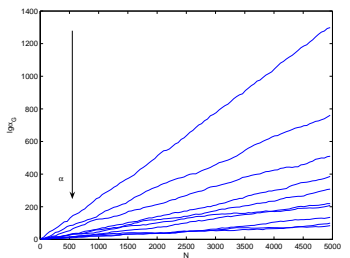

(b)

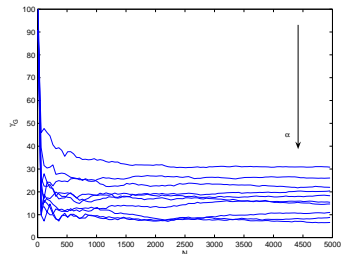

(d)
FIG. 6: The effect of $\alpha$ and size on symmetry of networks generated by the SLP model.(a) Simulation of SLP model with $\alpha$ varying from 0.1 to 1 in increment of 0.1 . The horizontal axis of the figure as well as two insets (a) and (b) is $\alpha$, the vertical axis of the figure is $\lg \alpha_{G}$, the vertical axis of the inset figure (a) and (b) are $\beta_{G}$ and $\gamma_{G}$, respectively. In the simulations, we use $n_{0}=10, \bar{m}=10, t=10000$ and employ two kinds of initial degree distributions. Blue square $(\square)$ shows an exponential distribution $P(m)=a \gamma^{-m}$ with $\gamma=3$; red circle(o) shows a power law distribution $P(m)=a m^{\gamma}$ with $\gamma=-1$. Figure (b), (c) and (d) show the growth of symmetry indices including $\lg \alpha_{G}, \beta_{G}$ and $\gamma_{G}(\%)$ of networks generated by SLP model. In the simulations shown in Figure (b), (c) and (d), a power law initial degree distribution is employed with $\bar{m}=10$ and $\gamma=-1$, we fix $n_{0}$ as 10 and vary $\alpha$ from 0.1 to 1 ( The arrow shows the direction of increasing $\alpha$ ). We vary $t$ from 0 to 5000 and capture the snapshots of the network every 50 units of time, thus we could get 100 samples of networks with linearly increasing sizes. Clearly, for all $\alpha$, the growth of automorphism group size $\alpha_{G}$ shows a obvious exponential trend; and decrease of $\beta_{G}$ shows an power law trend; and $\lim _{N \rightarrow \infty} \beta_{G}=\sigma(\alpha)$. When $\alpha$ varies from 0.1 to 1 , all three symmetry indices decrease. The inset of Figure (c) shows the amplified local plot, which clearly shows the fact that $\beta_{G}$ decrease with the growth of $\alpha$.

to have the structure of tree and it is desirable that tree tends to have higher degree of symmetry. Such result conforms to the result reported in [22] that $B A$ random trees and uniform random trees have higher degree of symmetry.

As shown in Figure $7(\mathrm{a})|7(\mathrm{~b})| 7(\mathrm{c})$, when $\langle k\rangle$ increase, the symmetry of the network rapidly decays to a constant level $\tau(\gamma)$, which is determined by the slope of the power law distribution. Obviously, the steeper the initial degree distribution is, the higher the symmetry level is. When $\gamma=0$, i.e., the slope of the double log distribution plot is zero, then symmetry of the network rapidly decays to zero or value close to 0 as $\langle k\rangle$ increases. However, with the slope becoming steeper, the symmetry of the network rapidly decays to an approximately constant value $\tau(\gamma)$ 


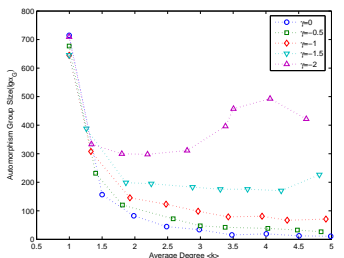

(a)

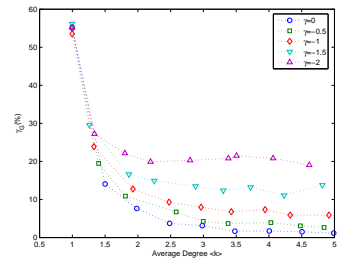

(c)

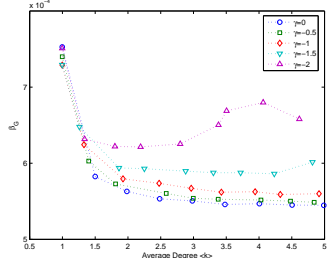

(b)

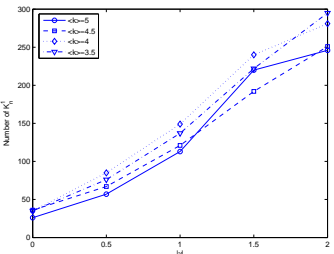

(d)
FIG. 7: The effect of average degree $\langle k\rangle$ on symmetry of networks generated by preferential attachment with initial degree following a power law distribution $P(m)=a m^{\gamma}$. In this simulation, we use $\alpha=1$ to eliminate the influence of similar linkage pattern, and other parameters are set as $n_{0}=10$, $t=5000$, and $\gamma=0,-0.5,-1,-1.5,-2$. For each $\gamma$, we vary $\langle k\rangle$ from 1 to 5 in increments of 0.5 . Figure (a),(b),(c) show the trend of automorphism group size $\lg \alpha_{G}, \beta_{G}$ and $\gamma_{G}(\%)$ with the growth of the average degree of the network, respectively. It is clear that symmetry of the network will rapidly (super linearly) decrease to an constant level for less steeper initial degree distribution. Figure (d) shows the relation between the number of local structure $K_{n, 1}$ and the slop $(|\gamma|)$ of the power law initial degree distribution for average degree as one of $\{5,4.5,4,3.5\}$. Parameters in Figure (d) are the same as Figure (a),(b) and (c). Clearly, with the increase of $|\gamma|$, more $K_{n, 1}$ will occur as the substructures of the network.

that is far larger than 0 as $\langle k\rangle$ increases. Thus, for steep log-log initial degree distribution, non-ignored degree of symmetry would be observed. Note that steeper initial degree distribution will result in a higher probability of smaller initial degree $m$, especially $m=1$; as a result, more tree-like symmetry will be found in the structure of the network. As shown in Figure 7(d), the number of $K_{n, 1}$ increases with the growth of $|\gamma|$; as observed in Table III the complexity and the size of $K_{n, 1}$ also increase with the slope of the double log initial degree distribution.

Thus, it's rational to conclude that only preferential attachment with initial degree following a distribution, will not necessarily reproduce symmetry of networks. Only in those cases that small initial degrees have higher probability of occurrences, especially $m=1$, will produce treelike symmetry of networks. To reproduce higher probability of smaller initial degree, we need to decrease the maximal initial degree or increase the slope of the initial degree distribution.

In summary, through the statistics of certain local symmetric motifs, such as (generalized) symmetric bicliques
TABLE III: Statistics of $K_{n, 1}$ in some SLP networks with power law initial degree distribution. All the parameters are set the same as Figure $7(\mathrm{~d})$ In this table, we records the number, the minimal, the maximal size of the maximal size of $\mathcal{K}_{n, 1}$ with $\gamma$ as one of $\{0,-0.5,-1,-1.5,-2\}$ and $\langle k\rangle$ as one of $\{3.5,4,4.5,5\}$.

\begin{tabular}{cccccc}
\hline \hline & \multicolumn{5}{c}{$\gamma$} \\
$\langle k\rangle$ & 0 & -0.5 & -1 & -1.5 & -2 \\
\hline 3.5 & $(35,2,4)$ & $(76,2,11)$ & $(137,2,10)$ & $(222,2,14)$ & $(295,2,26)$ \\
4 & $(35,2,5)$ & $(85,2,5)$ & $(149,2,6)$ & $(240,2,9)$ & $(281,2,41)$ \\
4.5 & $(36,2,4)$ & $(67,2,10)$ & $(121,2,9)$ & $(192,2,18)$ & $(251,2,47)$ \\
5 & $(26,2,4)$ & $(57,2,5)$ & $(113,2,6)$ & $(220,2,16)$ & $(246,2,40)$ \\
\hline \hline
\end{tabular}

in many real networks, we found that similar linkage pattern plays an important role in the origin of symmetry of networks. To incorporate this ingredient into BA model, we improved BA model in two aspects: (1) extending the initial degree from a constant value to a distribution; (2) increasing the linkage probability of those target nodes. Simulation shows that similar linkage pattern is responsible for the emergence of symmetry of networks, while preferential attachment with initial degree following a distribution will only reproduce tree-like symmetry in some cases.

Extensive existence of similar linkage pattern in real networks inspires us that behavior of individual nodes is far away from randomness, which provide us a brand new perspective, symmetry, to understand the selforganization of the complex systems. From this new viewpoint, in our studies we have found strong positive correlation between similar linkage pattern and symmetry of networks, which demonstrates that the emergence of complexity at macro system level is originated from the simple micro mechanism of individual component of the network system. Since symmetry breaking is the basic mechanism underlying the procedure of the network growth, we believe our studies will be of great value to help explore the laws dominating symmetry breaking in complex networks.

\section{ACKNOWLEDGEMENT}

The work was supported by the National Natural Science Foundation of China under Grant No.60303008; the National Grand Fundamental Research 973 Program of China under Grant No.2005CB321905 


\section{REFERENCES AND NOTES}

[1] R. Albert and A.-L. Barabási, Statistical mechanics of complex networks, Rev. Modern Phys. 74 (2002), no. 1, 47-97.

[2] R. Albert, H. Jeong, and A.-L. Barabási, Internet: Diameter of the world-wide web, Nature 401 (1999),130-131.

[3] L. A. N. Amaral, A. Scala, M. Barthélémy, and H. E Stanley, Classes of small-world networks, Proc. Natl. Acad. Sci. USA 97 (2000), no. 21, 11149-11152.

[4] A.-L. Barabási and R. Albert, Emergence of scaling in random networks, Science 286 (1999), no. 5439, 509-512.

[5] J. Kleinberg, Navigation in a small world, Nature 406 (2000), no. 6798, 845.

[6] Ch. Song, S. Havlin, and H.A. Makse, Self-similarity of complex networks, Nature, 433(2005), 392.

[7] M. E. J. Newman, The structure and function of complex networks, SIAM Rev. 45 (2003), no. 2, 167-256.

[8] D. J. Watts and S. H. Strogatz, Collective dynamics of small-world networks., Nature 393 (1998), no. 6684, 440442.

[9] R.Milo,S.Shen-Orr,S.Itzkovitz,N.Kashtan,D.Chklovskii, U.Alon,Network motifs: simple building blocks of complex networks ,Science 298(2002) no. 5594, 824-827

[10] M. E. J. Newman and M. Girvan, Mixing patterns and community structure in networks, Statistical Mechanics of Complex Networks, R. Pastor-Satorras, J. Rubi, and A. Diaz-Guilera (eds.), Springer, Berlin (2003).

[11] M. E. J. Newman, Mixing patterns in networks, Phys. Rev. E 67(2003),no.2, 026126.

[12] M. E. J. Newman,Assortative mixing in networks, Phys. Rev. Lett. 89(2002),no.20, 208701.

[13] M. E. J. Newman,Detecting community structure in networks, , Eur. Phys. J. B, 38(2004), 321-330.

[14] E. Ravasz, A. L. Somera, D. A. Mongru, Z. N. Oltvai, and A.-L. Barabási, Hierarchical organization of modularity in metabolic networks, Science 297 (2002), no. 5586, 1551-1555.

[15] B. D. McKay, Practical graph isomorphism, Congr. Numer. 30 (1981), 45-87.

[16] M.E.J.Newman, D.J.Watts , and S.H.Strogatz, Random graph models of social networks, Proc. Natl. Acad. Sci. U.S.A., 99(2002), 2566-2572.

[17] R.Ferrer.I.Cancho and R.V.Sol, The small world of human language. Proceedings of The Royal Society of London. Series B, Biological Sciences,268(2001), no. 1482, pp. 2261-2265.

[18] The CAIDA Group, The caida as

relationships

dataset,

2003-

2007 http://www.caida.org/data/active/as-relationships/

[19] J. Gehrke, P.Ginsparg, and J.M.Kleinberg. Overview of the 2003 kdd cup. SIGKDD Explorations, 5 (2003),no.2, 149-151.

[20] C. Stark, B.-J.Breitkreutz, T.Reguly, L.Boucher, A.Breitkreutz, and M.Tyers, BioGRID: a general repository for interaction datasets, Nucleic Acids Res. 34(2006),D535-D539.

[21] C.Godsil, G.Royle, Algebraic Graph Theory, volume 207 of Graduate Texts in Mathematics, Springer,2001.

[22] B.D.MacArthur, J.W.Anderson, Symmetry and self-organization in Complex Networks, arXiv:cond-mat/0609274, (2006).

[23] B.D.MacArthur; Snchez-Garca, J.Rubn; J.W.Anderson. Symmetry in Complex Networks, arXiv:0705.3215 (2007).

[24] V.Batagelj and A.Mrvar, Pajek - program for large network analysis, Connections, 21(1998), no. 2, 47-57.

[25] B. Bollobás, Modern graph theory, Graduate Texts in Mathematics, vol. 184, Springer-Verlag, New York, 1998.

[26] B. Bollobás, Random graphs, second ed., Cambridge Studies in Advanced Mathematics, vol. 73, Cambridge University Press, Cambridge, 2001.

[27] P.Erdös and A.Rényi, On random graphs, Publicationes Mathematicae(1959)290-297.

[28] which could be understood in the asymptotic way that the proportion of graphs on $n$ vertices that are asymmetric goes to 1 as $\mathrm{n}$ tends to $\infty$

[29] It may be confused between similar linkage pattern and assortative mixing. Both of the concept focus on the behavior of those nodes having similar properties. However, assortative mixing emphasize on inter-linkage between these nodes, while Similar linkage pattern only require that these nodes share similar linkage target, whether these nodes are inter-linked is not significant.

[30] which means that for each $v \in V_{1}, d_{K}(v)=d_{G}(v)$, where $d_{G}(v)$ is the degree of vertex $v$ in graph $G$

[31] Two subgraphs are non-disjoint implies that the node set of these two graphs are disjoint.

[32] Given automorphism partition group $(A u t(G), V)$ of graph $G=(V, E)$, we can get a partition $\mathcal{P}=$ $\left\{V_{1}, V_{2}, \ldots, V_{k}\right\}$ in the way that $x$ is equivalent to $y$ if and only if $\exists g \in \operatorname{Aut}(G)$, s.t. $x^{g}=y$. And each cell of the partition is called as an orbit of $(\operatorname{Aut}(G), V)$. An orbit is trivial if it only contains a single vertex, otherwise, the orbit is nontrivial.

[33] In our study, for the convenience of denotation, the average degree $\langle k\rangle$ is defined as $\frac{M}{N}$, where $N$ and $M$ are the numbers of nodes and edges, respectively. Obviously, $\langle k\rangle$ is a half of the actual average degree. 